\title{
Special issue on multimedia in technology enhanced learning
}

\author{
Zhihan $\mathrm{Lv}^{*}$ \\ SIAT, Chinese Academy of Science, Shenzhen, China
}

The advent of new technologies such as Multimedia and the Internet of Things has brought opportunities for Technology Enhanced Learning. These technologies can be applied in intelligent learning functions, which range from Smart Schools, Smart Classes to Smart Learning at Home and so on. Assessment and evaluation methods need to be developed and involved into the iterative designing processes. This new notion involves some tasks in which traditions are replaced by multimedia technology requiring manual discrimination and resolution to reach optimization. In addition, with the development of Internet of Things, Technology Enhanced Learning application pays greater attention to the interconnection between multi-modal interactive technologies and information systems to the maximum extent possible. Increased networking in different scales from global to local is having a profound effect on learning and teaching. It makes new forms of collaborative and personalized learning experiences a reality. Then it can be seen that further efficient management and deeper analysis of Technology Enhanced Learning are the key tasks in developing smart schools or individual learning applications at home based on Multimedia technologies.

For this special issue we selected 26 manuscripts from a total of 60 submissions after a careful peer review process. A call-for-papers was advertised in

${ }^{*}$ Corresponding author. Zhihan Lv, SIAT, Chinese Academy of Science, Shenzhen, China. Tel./Fax: +86 13791802272; E-mail: 1vzhihan@gmail.com. the main scientific communities related to the topic of this special issue and also distributed at world-leading research groups such as at the University of Oxford, University College London, Oak Ridge National Laboratory, Peking University, KTH Royal Institute of Technology, French National Centre for Scientific Research (CNRS), National Institutes of Health. The accepted manuscripts range from countries as diverse as the USA, UK, China, Sweden, France, Saudi Arabia, Pakistan and Palestine among others. Each manuscript selected was blind reviewed by at least three reviewers. It's worth to mention that in this issue we publish the paper Towards the Design of Effective Freehand Gestural Interaction for Interactive $T V$, which is an extended version of the paper that won the best paper award at EuroITV/ACMTVX'13.

The first paper comes from the School of Electronic, Electrical and Systems Engineering, University of Birmingham, UK. Tracking is a fundamental problem for event recognition. Particle filter (PF) is acknowledged for its efficiency in dealing with multi-modal visual tracking problem of general nonlinear and non-Gaussian system. It therefore emerged as an appealing tool for tracking objects in video sequences. Although many proposals have been put forward to deal with various scenarios and enhance $\mathrm{PF}$ convergence properties, it is acknowledged that comprehensive evaluations of the proposals are still lacking. 'Performance Evaluation of Particle Filter Based Visual Tracking' aims to contribute to this ongoing research. Especially, simulated 
videos were created to analyze the influence of the target appearance according to various noise intensities that entails partial or full occlusion scenarios. In the simulated videos, the experiment provided more accurate conclusions given the range of involved factors, w.r.t target model, similarity measurement, and environmental distraction. To validate the conclusion from the simulated videos, the experiment was also conducted in the benchmark videos.

The second paper comes from West Virginia University, USA. Multimedia has changed traditional white-blackboard lecture forever and reformed continually the forms and presentation of materials. 'An Online Marking System Conducive to Learning' proposes an online marking system. The realization of this system will help improve working efficiency of marking papers, reduce potential cheating and facilitate the teacher storage and analysis students' examination papers. The system is realized based on B/S structure (Browser/Server structure) which will increase the flexibility of online marking as long as the teacher accesses to the Internet. The system has realized online marking and the storage streamlined, which will improve teacher's work efficient and provide a reference for learning schedule and a feedback to teaching achievements.

\section{'Multimedia security in laboratory system based on} cloud platform' is from West Virginia University, USA. The system contains records of daily reports, papers, patents, projects and multimedia files storage function. Compared with existing laboratory systems, the system focuses on needs of graduate students in laboratory, replaces equipment management and fund management of traditional laboratory for daily reporting and scientific research of graduate students in laboratory. The system is built on cloud platform. A set of systems are set to meet demands of graduate students in laboratory, so as strengthen communication between teachers and students and strengthen exchange among students, as well as improve the quality of postgraduate training. Therefore, this system conforms to the development trend of technology, and the combination of cloud computing technology becomes preliminary exploration for the development of laboratory management system.

\section{'A Robust Energy-Efficient Routing Algorithm to Cloud Computing Networks for Learning' is from University of Oxford, UK. With the advancement of multimedia, Internet of things, and cloud computing technologies, technology enhanced learning appli-}

cations such as smart school and smart learning at home have been paid more attentions to. Cloud computing services, which provide a novel collaborative and personalized learning style, have an important impact on learning and teaching. However, with the rapid development of cloud computing, the energy consumption problem for cloud computing networks cannot be ignored and the idea of energy efficiency comes into being. Currently, the main method to solve energy consumption problem is to turn the idle routers and links into sleeping, but this approach cannot be suited for cloud computing networks and may bring down network resource utility and network performance. The paper combining the Sleeping Redundant Links Algorithm (SRLA) with the Minimum Criticality Routing Algorithm (MCRA), proposes a Robust Energy Efficiency Routing Algorithm (REERA) used for cloud computing networks. Comparing REERA with OSPF-based algorithm, the simulation results show that REERA outperforms Open Shortest Path First (OSPF) -based algorithm, and REERA can realize the energy efficient routing algorithm under the premise of network robustness constraints.

\section{'Dynamic Access Approach to Multiple Channels in Pervasive Wireless Multimedia Communications for Technology Enhanced Learning' is called from} University of Minnesota, USA and University of Maryland, Baltimore County, USA. With the development of multimedia and Internet of things technologies, technology enhanced learning applications such as smart class and smart learning at home have received more attentions from industrial and academic communities. However, pervasive wireless communications, which build new collaborative and personalized learning patterns, have an important impact on learning and teaching. For current pervasive wireless networks, spectrum resources become increasing lack due to the introduction of a large number of new wireless technologies and thus the access of many new devices to wireless networks for all kinds of applications such as online smart learning. How to exploit effectively spectrum resources in current pervasive wireless networks is a larger challenge. This paper analyzes more complex multiple channel model with multiple primary and secondary users. It exploits the lognormal distribution to characterize primary user behaviors and use opportunistic spectrum access to obtain the sensing status of channels. Simulation results show that the approach is feasible and promising. 
'Evaluation of three-dimensional computer visual materials to support user's participation in architectural design process' is a collaborative work between Wuhan University, China, An Najah National University, Palestine and Multimedia University, Malaysia. This paper evaluated the use of Three-dimensional (3D) Computer Visual Materials (CVM) to support user participation in the early design stages for a private house. A qualitative approach was identified in two stages. Firstly, a semi-structured interview with a sample of architectural firms was conducted, and secondly, data was collected from conversational dialogues between an architect and a list of clients. The results revealed that various stages of the design process have different responses with respect to the use of Visual Material (VM) and its impact on user participation. In addition, there appeared to be a direct relationship between the Level of Details (LoDs) and specific design stages.

As an ancient character of larruping fascination, Nvshu has aroused international scholars' interest, and the research of Nvshu has been developed widely. However, the research of Nvshu mostly based on handwriting at present has been developed slowly. So informationization of Nvshu has stared us in the face. The standardization of Nvshu is researched in 'Study on Algorithm and Application to Radical Input Method and Standardization of Nvshu'. Based on research of standardization, according to the research of literacy psychology and fuzzy psychology, the relationship of character recognition of Nvshu and computer input method was illuminated, and the samples of the Nvshu by using the statistic method of clustering were analyzed, in order to extract the common parts of the Nvshu characters to form the etymons, which were the basic of computer input method of Nvshu. This input method is accurate, stable, easily study and easily operable. Meanwhile this input method would make outstanding contributions to the protection of ancient literature.

\section{'User Authority Ranking Models for Community}

Question Answering' is a collaborative work by Sun Yat-sen University, The Hong Kong Institute of Education, City University of Hong Kong and Caritas Institute of Higher Education, China. The proliferation of knowledge-sharing communities has generated large amounts of data. Prominent examples of how user-generated content can be harnessed include IBM's Watson question answering system and Apple's Siri, the question answering application in iPhones. Facing such massive data, user authority ranking is important to the development of question answering and other e-commerce services. The authors propose three probabilistic models to rank the user authority of each question. Compared to the existing approaches focused on the user relationship primarily, the proposed method is more effective because the link structure and topical similarities between users and questions are considered simultaneously. Experimental results show that the proposed model outperforms other baseline methods in ranking the user authority.

\section{'A Hierarchical Model to Learn Object Proposals} and Its Applications' is from Computer Science Department, Utah State University, USA. Generating class-agnostic object proposals followed by classification has recently become a common paradigm for object detection. Current state-of-the-art approaches typically generate generic objects, which serve as candidates for object classification. Since these object proposals are generic whereas the categories for classification are domain specific, there is a gap between the generation of object proposals and the classification of object proposals. In the paper, by taking advantages of the intrinsic structure and the complexity of each category of objects, it proposes a novel tree-based hierarchical model to learn object proposals, from top proposals produced by the existing object proposals generation methods. Experiments demonstrate the significant improvement of the proposed approach over the state-of-the-art method in terms of object detection rate. An application is proposed based on this approach to help children learn and recognize objects by their visual appearances and their sub-parts structures.

\section{'A new robust color image watermarking method for multimedia technology enhanced learning pro-} tection' is from Pennsylvania State University, USA. In multimedia technology enhanced learning, digital watermarking is an important technique for protecting copyrighted multimedia materials. This paper proposes a robust color image watermarking scheme using quaternion geometric Legendre moment invariants (QGLMIs). Firstly, the original image is divided into color image blocks. Then, the Quaternion Discrete Cosine Transform (QDCT) is performed on the color image block. Finally, the digital watermark is embedded into original color image by adaptively modulating the real QDCT 
coefficients of color image block. For watermark detecting, the LS-SVM correction with low-order QGLMIs is utilized. Experimental results show that the proposed color image watermarking is not only invisible and robust against common image processing operations (especially for color attacks), but also robust against geometrical distortions.

\section{'Inferring popular locations in urban for professi-} onal education' is from Huazhong University of Science and Technology, China. With the rapid development of urbane-centered economy, urban area has gone through strong but heterogeneous sprawl. In such complex urban systems, it is impossible to established teaching centers of night school in every district of city for continuing education programs. Part-time students tend to be educated in popular locations of city due to convenience. Since call logs and geographical nature of mobile phone data can provide an opportunity to measure human behavior and social dynamics, this paper investigates how to infer urban popular locations with large-scale quasi-social network for avoiding the limitation of data collection and even privacy problems. A large-scale quasi-social network model is developed via measuring the number of shared-user between zones, which is different from previous models for social network.

Visual tracking is of great importance in multimedia technology enhanced learning. Many humanmachine-interaction based learning/teaching activities need tracking of specific object. Particle filter has grown to be a standard framework for visual tracking in the past decades. One of its key issues is the design of the proposal distribution which can greatly affect the performance of particle trackers. 'Tracking Objects in Video-based Education Using an Enhanced Particle Filter' propose an enhanced particle filter for robust visual tracking. Experimental results show that the proposed tracking algorithm is better than or not worse than several other tracking algorithms over several public sequences.

'Drift Robust Non-rigid Optical Flow Enhancement for Long Sequences' is a collaborative work by University College London, UK, University of Bath, UK and Google headquarters, USA. It is hard to densely track a nonrigid object in long term, which is a fundamental research issue in the computer vision community. This task often relies on estimating pairwise correspondences between images over time where the error is accumulated and leads to a drift. This paper introduces a novel optimisation framework with an Anchor Patch constraint. It is supposed to significantly reduce overall errors given long sequences containing nonrigidly deformable objects. The work demonstrate the success of the proposed approach by showing significant error reduction on 6 popular optical flow algorithms applied to a range of realworld nonrigid benchmarks. The work also provides quantitative analysis of the approach given synthetic occlusions and image noise.

'Telepresence Mechatronic Robot (TEBoT): Towards the Design and Control of Socially Interactive Bio-Inspired System' is from Umea University, Sweden and KTH Royal Institute of Technology, Sweden. Socially interactive systems are embodied agents that engage in social interactions with humans. From a design perspective, these systems are built by considering a biologically inspired design (Bio-inspired) that can mimic and simulate human-like communication cues and gestures. The proposed socially interactive bio-inspired system is based on an intuitive integration-design strategy that combines computer vision based geometric head pose estimation algorithm, model based design (MBD) approach and real-time motion planning techniques. The authors have conducted an extensive testing to demonstrate effectiveness and robustness of the proposed system.

'Towards a Resource Migration Method in Cloud Computing Based on Node Failure Rule' is from Peking University, China and CNRS, France. The virtualization design management and adaptive resource migration techniques are typically used for energy saving and system reliability improvement in the cloud computing system. This paper presents a resource migration technique based on node performance and failure rule (NPFR-RMT). It uses Broker to access node performance to determine abnormal nodes and failure nodes. Comparison experiment results show that the NPFR-RMT method presented in the paper can achieve expected aim that reducing energy consumption of data centers while ensuring service performance.

'Adaptive cutoff distance: clustering by fast search and find of density peaks' is a collaborative work by Beijing Normal University, China, University of Management Sciences and Information Technology, Pakistan, and University of Engineering and Technology, Pakistan. Clustering by fast search and find 
of density peaks (CFSFDP) was proposed to create clusters by finding high-density peaks, quickly. This paper proposes an adaptive way to estimate the accurate $\mathrm{Cd}$ by using the characteristics of Improved Sheather-Jones (ISJ) method named as IJS-CFSFDP. ISJ method provides the best estimation for $\mathrm{Cd}$ to measure accurate density of each data point. The authors perform a number of experiments on standard benchmark clustering datasets and real academic dataset of students. The evaluated clustering results on education dataset validate the IJS-CFSFDP can be used to make intelligent contents delivery system based on the capability and intelligence of the student. The experimental results on synthetic datasets show that the proposed adaptive $\mathrm{Cd}$ method creates better clusters as compare to the CFSFDP, mean shift, affinity propagation and k-means.

Foggy weather brings lots of inconvenience for outdoor safety surveillance in the densely populated school education area. Research on image and video dehazing is able to solve this problem. Most existing methods recover the haze-free scenes relying on the atmospheric scattering model in image dehazing, which often suffer from halo artifacts because of the indistinct edges in the scene depth map. L0 gradient minimization is introduced to better preserve and locate important edges globally to optimize the scene depth map, making use of this physical model in 'Depth estimation for image dehazing of surveillance on education'. Firstly, a rough scene depth map based on the inherent boundary constraint prior on the scene is estimated. Secondly, the rough scene depth map in bright regions is compensated with an adaptive term. Then this compensated scene depth map is put into an optimizing framework to get a refined depth map to make it closer to the ideal scene depth. Finally, with the refined depth map and global atmospheri c light, it can recover the haze-free scenes using the atmospheric scatting model. Experimental results show the proposed is better to obtain haze-free scenes with sharp edges, abundant details and vivid color while dealing well with bright areas.

'Style-Sensitive3D ModelRetrievalthrough SketchBased Queries' is from Oak Ridge National Laboratory, USA. Traditional sketch-based 3D model retrieval methods are content-based, which return the search results by ranking the geometric similarities among a free-hand drawing and 3D model candidates. These conventional methods do not consider personal drawing characteristics and styles (abbreviated as styles), which are obvious and important in user's sketch queries. An ordinary user presumably is not a professional and skillful artist. Therefore, users are likely to introduce personal drawing style in sketching 3D model rather than faithfully render the model according to its geometric perspectives. For amateurs, such personal styles are unintentionally introduced due to their limited sketching capabilities. As determined by a person's sketching habit, personal drawing styles are largely personally consistent and stable. Ignoring such non-trivial personal styles while attempting to reconstruct intended models according to their sketch inputs does not usually produce satisfactory outcomes, in particular, for amateur sketchers. To overcome this problem, this paper proposes a novel style-sensitive 3D model retrieval method based on three-view user sketch inputs. The new method models users' personal sketching styles and constructs joint tensor factorization to improve the retrieval performance.

\section{'Forensic Artifacts Modeling for Social Media Client Applications to Enhance Investigatory Lear-} ning Mechanisms' is from King Saud University, Saudi Arabia. Since the inception, social media have emerged as an inexpensive and efficient way of communication. Facebook is leading the global social networking that provides multiple channels for sharing information with the community. There are various ways to access a Facebook account such as through a web browser, mobile applications and third-party desktop applications for Facebook. Platforms like desktop applications for social media are of benefit to users as they provide ease of access and availability, but in the case of a user's data privacy breach including illegitimate social behavior, it becomes a challenge for the forensic examiners to track such activities for learning purposes. The aim of digital forensics is to get hold of the existing legal evidence present within the digital media. The major focus of this paper is to investigate and analyze the two Facebook desktop applications: Fosimo and Sobees, with respect to the tracking of user activities. The Facebook desktop applications are examined by analyzing the Windows registry, browser, cookie, cache, connection analysis and the local installation directory. As a result of this research, this work ensures that the footprint gathering of the user activity from Windows registry and browser files is performed in such a way that it could be used for forensic artifacts modeling keeping the integrity of the whole process. 
'Towardsthe Design of Effective Freehand Gestural Interaction for Interactive $\boldsymbol{T V}^{\prime}{ }^{\prime}$ is a collaborative work by Xiamen University of Technology, China, University College London, and University of Bath, UK. This paper is the extended version of a paper that won best paper award at EuroITV/ACMTVX'13. As interactive devices become pervasive, people are beginning to looking for more advanced interaction with televisions in the living room. Interactive television has the potential to offer a very engaging experience. But most common user tasks are still challenging with such systems, such as menu selection or text input. And little work has been done on understanding and supporting the effective design of freehand interaction with a TV in the living room. This paper performs two studies investigating freehand gestural interaction with a consumer level sensor, which is suitable for TV scenarios. The first study investigates a range of design factors for tiled layout menu selection, including wearable feedback, push gesture depth, target size and position in motor space. The results show that tactile and audio feedback have no significant effect on performance and preference, and these results inform potential designs for high selection performance. The second study investigates a common TV user task of text input using freehand gesture. Results show that ease of use and error tolerance can be both achieved using a text entry method utilizing a dual circle layout and an expanding target selection technique. Finally, the authors propose design guidelines for effective, usable and comfortable freehand gestural interaction for interactive TV based on the findings.

\section{'A Novel 3D Model Retrieval System Based on} Three-view Sketches' is from Oak Ridge National Laboratory, USA. 3D models can be used in 3D printing and many other areas. At present, there are a lot of researches on 3D model retrieval and sketch is considered to be important for 3D model retrieval. This paper develops a new 3D model retrieval prototype system based on style-sensitive 3D model retrieval method and three-view user sketches. It also implements user-friendly graphic interfaces for the 3D model retrieval system. The paper explores the performance of the system by conducting a series of 3D model retrieval experiments on the Princeton shape benchmark data set. Experimental results show that the new retrieval system can obtain satisfactory retrieval results. And the results of the new method are superior to some content-based 3D model retrieval methods, in terms of both quantitative search performance metrics and qualitatively measured user search experiences.

\section{'Topologies for combining the Internet of Things} and Serious Games' is from Liverpool John Moores University, UK. Serious Games have been established over recent years as a means of utilising gaming for applications other than entertainment. With the emergence of the Internet of Things (IoT) paradigm, a new direction for serious games arises, where data gathered from the physical environment can be utilised towards new novel applications. This paper presents findings from extensive research into IoT, Serious Games, Pervasive Games and Gamification, IoT topologies and Wireless Sensor Networks (WSN), to identify the requirements of a topology for Serious Games and IoT. By understanding the topological requirements for combining IoT and Serious Games, the development process is reduced, allowing for the advancement in the mentioned field. Three topologies are presented for combining IoT with Serious Games and a detailed topology for developing a Serious Game that monitors student attendance is presented. Also included, is an insight into the new paradigm of Smart Serious Games (SSGs). This paper will aid future research and development in SSGs determine effective network topologies.

\section{'Accurate RFID Localization Algorithm with Parti-} cle Swarm Optimization Based on Reference Tags' is a collaborative work by Shenzhen University, China and The Hong Kong Polytechnic University, China. RFID technology has been widely used for object tracking in indoor environment due to their low cost and convenience for deployment. Existing RFID localization approaches rely on signal strength to measure the distance between RFID reader and tags. However, because of the environmental complexity and inferences, the measurement of distance by signal strength is not accurate, which causes large error in localization. This paper develops a novel algorithm to improve the RFID localization accuracy. The result shows that compared with the previous method without the correction factor, the proposed approach can increase the accuracy by $50 \%$. This method has good application prospect in medical equipment management.

'An Efficient Estimation Method Coping with the Capture Effect for RFID Tags Identification and Application in Remote Learning' is from Xiamen 
University, China and Ulster University, UK. In telecommunications, the capture effect is a phenomenon associated with frequency modulation reception in which two strong signals at, or near, the same frequency or channel will be demodulated. In wireless networks, a frame collision does not necessarily result in all the simultaneously transmitted frames being lost. Depending on the relative signal power and the arrival time of the involved frames, one frame can survive the collision and be successfully received by the receiver. Efficient object identification with passive RFID tags. In former works, many researches focus on the RFID anti-collision protocol only, however, less did pay special attention to the capture effect mainly to keep the design simple and cost low. Nonetheless, the capture effect occurs frequently in real deployments. This paper has taken the capture effect into consideration and combined this thought to an efficient RFID anti-collision protocol, then proposed an efficient method to estimate the optimal frame length to adjust the quantity of RFID tags.

\section{'An Improved Hash-Based RFID Two-WaySecurity Authentication Protocol and Application in Remote} Education' is another paper from Xiamen University, China and Ulster University, UK. With the increasingly expanding application of RFID technology, the security and privacy issues of RFID system has also been a widespread concern. The existing RFID security protocol can't simultaneously meet the requirements of low-cost, low computing, high efficiency and high security. In this paper, through the analysis of common principles and shortcomings of RFID security protocols based on Hash Function, this paper improve the security protocols, so as to achieve the objectives of two-way authentication. This paper theoretically proves the protocol security through BAN logic. And the new protocols can effectively solve the security and privacy problems such as replay attack, fake attack, location privacy, anonymous tags and so on. The paper proposes a kind of authentication and communication security mechanism that makes full use of the functions of the conditional Access Module (CAM) which exist in the original remote education system and when authenticating the nodes of the system, the authors use the proposed security scheme. Analysis results showed that the mechanism is safe, reliable, strong compatibility, economic and applicable.
'Identifying the influential spreader in multilayer interaction online social networks' is a collaborative work by University of Malaya, Malaysia and Xi' an Jiaotong Liverpool University, China. Online social networks (OSNs) portray a multi-layer of interactions through which users become a friend, information is propagated, ideas are shared, and interaction is constructed within an OSN. Identifying the most influential spreaders in a network is a significant step towards improving the use of existing resources to speed up the spread of information for application such as viral marketing or hindering the spread of information for application like virus blocking and rumor restraint. Users communications facilitated by OSNs could confront the temporal and spatial limitations of traditional communications in an exceptional way, thereby presenting new layers of social interactions, which coincides and collaborates with current interaction layers to redefine the multiplex OSN. In this paper, the effects of different topological network structure on influential spreaders identification are investigated. The results analysis concluded that improving the accuracy of influential spreaders identification in OSNs is not only by improving identification algorithms but also by developing a network topology that represents the information diffusion well. Moreover A topological representation is proposed which takes into accounts both multilayers interactions as well as overlaying links as weight. The results show the proposed topological representation has given more reliable measurement compared to single layers representation.

\section{'Study on FOA_BP Remote Sepsis Diagnosis Based} on Wireless Sensor Network' is called from Institutes of Health, USA. With the development of economic level and growth in the living standard, how to enhance the accuracy and efficiency of remote sepsis diagnosis has become the key issue and hot topic in the current medical research. As the traditional BP neural network is poor in the generalization ability and needs a large number of samples, this paper proposed the study on remote sepsis diagnosis by using the FOA-optimized BP neural network. The FOA-BP algorithm is significantly superior to the BP neural network algorithm in the diagnosis accuracy, verifying the validity and reliability of making a sepsis diagnosis with FOA_BP. 
'Self-education of Agents in the Multi-Airport Logistics System: A Multiple Cases Study' is a collaborative work by Nanjing University of Aeronautics and Astronautics, China, Civil Aviation University of China, China and Florida Institute of Technology, USA. The Multiple Airports System (MAS) has been the topic of an increasing number of studies over recent years in the spatial and network analysis. However, there is relatively limited literature on the MAS in the perspective of logistics. This paper proposes the concept of the Multi-Airport Logistics System (MLS) by reviewing the related literature on MAS and comparing the performance of cargo and passengers transport in the U.S. MASs. By using Multiple Cases Study, it found that the agents gain experiences from the interaction with other agents and the environment, i.e. self-education of Agents by building blocks in the system. 